\title{
ESTUDO DA SUSCETIBILIDADE "IN VITRO" A UM NOVO ANTIMICROBIANO (IMIPENEM) DE PATÓGENOS ISOLADOS DE PACIENTES HOSPITALARES EM VÁRIOS CENTROS
}

\begin{abstract}
RESUMO
O imipenem é um novo antibiótico Beta lactâmico, carbapenêmico, altamente potente e com amplo espectro de atividade antimicrobiana. Com intuito de compro var a eficácia "in vitro" deste fármaco em patógenos mais frequentes em nosso meio, descrevem os autores, os resultados das provas de suscetibilidade por discos e/ou a correspondência por provas de diluição para determinação da concentração inibitória mínima (CIM) em 1230 cepas compreendendo 41 diferentes espécies bacterianas recém-isoladas, principalmente de pacientes hospitalares em 5 diferentes centros médicos de São Paulo, Rio de Janeiro e Salvador. Nossos resultados preliminares com o antibiótico, em fase final de experimentação clínica e laboratorial, em nosso meio, foram muito promissores, com $96.79 \%$ de cepas suscetiveis pela prova do disco (10 $\mu \mathrm{g}$ de imipenem) e $92,31 \%$ de correspondência pela determinação do CIM (concentraçōes de até $4 \mu \mathrm{g} / \mathrm{ml}$ ).

Das 9 espécies bacterianas mais frequentemente isoladas, correspondendo a $1008(82 \%)$ das 1230 cepas de nosso material, as sensibilidades pela prova do disco foram de 99\% (E. coli), 93\% (Pseudomonas aeruginosas), 87\% (Staphylococcus aureus), $100 \%$ (Klebsiella pneumoniae), $98 \%$ (Klebsiella sp) e $100 \%$ (Streptococcus faecalis) com boa correspondência pela determinaçāo do CIM até $8 \mu \mathrm{g} / \mathrm{ml}$; e $100 \%$ para o anaeróbio Bacteróides sp (CIM até $4 \mu \mathrm{g} / \mathrm{ml}$ ). Ressaltam os autores a eficácia "in vitro" contra patógenos hospitalares que apresentam elevados índices de resis tência à grande maioria de antibióticos como o Pseudomonas aeruginosa e para anaeróbios, notadamente o Bacteróides sp.
\end{abstract}

UNITERMOS: Antimicrobiano; Imipenem; Infecçōes hospitalares.

(1) Professor da Disciplina de Patologia Clinica Faculdade de Medicina da Universidade de São Paulo. Chefe, Laboratório de Investigaçāo Médica de Bacteriologia - HC-FMUSP e Instituto de Medicina Tropical de São Paulo - FMUSP. Sảo Paulo, SP, Brasil.

(2) Professor Assistente da Disciplina de Patologia Clinica da Faculdade de Medicina da Universidde de Sāo Paulo. Chefe, Seçảo de Bacteriologia, Laboratório Central - HC FMUSP. Sāo Paulo, SP. Brasil.

(3) Professor da Disciplina de Microbiologia Faculciade de Ciencias Médicas da Santa Casa de Sāo Paulo. Săo Paulo, SP, Brasil.

(4) Professora da Disciplina de Microbiologia Faculdade de Medicina da Universidade Federal da Bahia. Salvador, Bahia, Brasil.

(5) Professor da Disciplina de Microbiologia e Imunologia - Faculdade de Ciências Médicas da Universidade do Estado do Rio de Janeiro. Rio de Janeiro, RJ, Brasil

(6) Professor da Disciplina de Microbiologia - Faculdade de Medicina da Universidade Federal do Rio de Janeiro. Rio de Janeiro, RJ, Brasil

Endereço para correspondència: Dr. Cid Vieira Franco de Godoy. Laboratório de Investigaçăo Médica e Bacteriologia do Instituto de Medicina Tropical de Sáo Paulo. Av. Dr. Enéas de Carvalho Aguiar, 470. CEP 05403 São Paulo, SP, Brasil. 
GODOY, C. V. F. de; MENDES, C. M. F.: MimiçA, I.: Oliveira, M. de; SuAssunA, I. \& UzEDA, M. de - Estudo da suscetibilidade "in vitro" a um novo antimicrobiano (IMIPENEM) de patógenos isolados de pacientes hospitalares em vários centros. Rev. Inst. Med, trop. S. Paulo, 31(3): 169.176, 1989.

\section{INTRODUÇÃO}

O imipenem é um derivado da Tienamicina, um novo antibiótico beta-lactâmico produzido por fermentação pelo microorganismo do solo Streptomyces cattleya (KAHAN \& col.). A Tie namicina é o primeiro representante de uma nova classe de antibióticos, os carbapenémicos, com características estruturais únicas que o di ferenciam de todos os demais antibióticos betalactámicos nàturais ou sintéticos previamente descritos (ALBERS-SCHONBERG \& col.). E dotada de potente atividade antimicrobiana contra um amplo espectro de bactérias, contudo sua utilidade prática é prejudicada pelo alto grau de instabilidade em estado sólido e, mesmo em altas concentraçōes, quando em solução. A ob tenção por síntese de um derivado amidínico, o $\mathrm{N}$-formimidoyl tienamicina levou a composto cristalino, estável - o imipenem (LEANZA \& col.), o primeiro antibiótico derivado da tiena micina de uso clínico. Estudos iniciais da atividade antimicrobiana do imipenem demonstraram acentuada atividade bactericida "in vitro" e grande eficácia de proteção em infecçōes expe rimentais contra vários patógenos, incluind o ce pas portadoras de resistência mediada por beta lactamases para penicilinas e cefalosporinas (KROPP \& col., 1980).

Estudos comparativos mostram que, com raras exceções, a potência e espectro do imipe nem excedem a de outros antibióticos beta-lac. tâmicos tanto contra espécies gram positivas, incluindo os enterococcus, como espécies gram negativas, entre os quais a Pseudomonas aeruginosa (KESADO \& col.) e anaeróbios (WEXLER \& FINEGOLD).

Atualmente numerosos estudos foram publicados referentes a suscetibilidade bacteriana ao imipenem, compiladas na revisão de KROPP \& col., 1985.

O arsenal terapêutico antimicrobiano atual mente disponivel conta com antibióticos que in dividualmente possuem atividade contra várias espécies de bactérias patogênicas e que quando considerados em grupo compreendem atividade conjunta para quase todos os patógenos. A nosso ver a pesquisa de novos antibióticos com características e atividades peculiares pode ser justifi cada para as seguintes circunstâncias: no trata mento de certas infecçōes por estafilococos resis tentes à meticilina ou oxacilina; por certas bactérias gram negativas para terapeutica inicial presuntiva em infecçōes muito graves, incluindo as que ocorrem em pacientes neutropênicos ou imunocomprometidos; para o tratamento de infecções "profundas" como as que podem ocor rer associadas a implantes; e para o tratamento de infecçós mistas, causadas por duas ou mais espécies bacterianas - aeróbicas ou anaeróbicas - como ocorre na bacteriemia polimicrobiana e sepsis abdominal. Apresentando pois, pela literatura científica, o imipenem algumas características para as intercorrências acima descritas, pareceu-nos oportuno e justificado um estudo em nosso meio da suscetibilidade bacte riana a este antibiótico.

Com este objetivo foram selecionados centros de hospitais universitários de 3 capitais do país: São Paulo, Rio de Janeiro e Salvador e realizadas provas de suscetibilidade ao imipenem com cepas de bactérias patogênicas recémisoladas abrangendo as mais freqüentemente associadas a infecçōes correntes, com ênfase àque las provenientes de pacientes hospitalizados.

\section{MATERIAL E MÉTODOS}

Cepas de patógenos bacterianos: 1230 pató genos recém-isolados de igual número de pacientes, sendo a maioria de hospitalizados, portadores de infecçōes de média a severa gravidade. e uma outra parte constituida por pacientes ambulatoriais com infecçōes de pouca gravidade.

A proveniência dos 1230 pacientes incluídos neste estudo obedeceu à seguinte distribuiçāo:

Hospital das Clínicas, Faculdade de Medicina da USP

Hospital da Santa Casa de Misericórdia de São Paulo

Hospital das Clínicas da Faculdade de Medicina da UERJ

Hospital das Clínicas da Faculdade de Medicina da UFRJ

Hospital das Clínicas da Faculdade de Medicina da UFB
513 


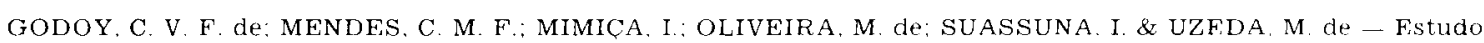
da suscetibilidade "in vitro" a um novo antimicrobiano IMIPENFM d de patogenos isolados de pacientes hospitalares em vários centros. Rev. Inst. Med. trop. S. Paulo, 31(3): 169 176, 1989.

Destes foram coletadas amostras clínicas apropriadas para cultivo em meios aeróbios ou anaeróbios compreendendo diversas fontes bio lógicas, a saber: sangue, urina, fezes. líquido cé falo raqueano, liquido ascítico, liquido pleural, bile, lavado brónquico, aspirado transtraqueal. raspado de osso, pele, peças cirúrgicas, zaraga toa de orofaringe e nasal, secreçōes clinicas ci rúrgicas diversas como: secreção uretral, secre ção vaginal, produto de drenagem de abcesso, entre outros

As culturas e identificaçōes dos patógenos isolados foram realizados por técnicas bacterio lógicas clássicas e as cepas foram recultivadas previamente às provas de suscetibilidade para garantia de tratar-se de cultura pura.

Agentes antimicrobianos: Imipenem em frascos de $50 \mathrm{mg}$ do sal N Formimidoyl Tiena micina cristalizado. com poténcia $98.9 \mu \mathrm{g} \mathrm{mg}$. pe so molecular 317.35 , lote $638.59601 \mathrm{D} 562$. forne cido por Merck Sharp \& Dohme Research Laboratories; discos de papel de filtro com a potencia declarada de $10 \mathrm{mg}$ do imipenem, lote $4 \mathrm{~A} 051$ (Merck) e discos de papel de filtro com antibió ticos usuais obtidos no comércio (Cefar e Difco).

\section{Provas de suscetibilidade}

Foram preparadas do imipenem liofilizado soluçōes apropriadas com tampāo fosfato 0,01 M, logo antes do uso. As cepas a serem testadas foram cultivadas em caldo Mueller-Hinton e ajustadas apropriadamente para fornecer apro. ximadamente $4 \times 10^{5} \mathrm{CFU} / \mathrm{ml}$ (WASHINGTON II \& SUTTER).

A determinação de concentração inibitória minima (CIM) foi definida como a menor concen traçāo antibiótica resultando na ausência de crescimento visivel após 18 a 24 hs de incubaçāo a $37^{\prime \prime} \mathrm{C}$ (BARRY \& THORNBERRY; SUTTER \&.WASHINGTON II: KROPP \& col.; WEXLER \& FINEGOLD). Considerou-se como padráo in terpretativo CIM: $4 \mu \mathrm{g} / \mathrm{ml}=$ sensivel; 5 a $8 \mu \mathrm{g} / \mathrm{ml}$ = intermediário e $9 \mu \mathrm{g} / \mathrm{ml}=$ :esistente ( $\mathrm{SHUN}$ GU \& col.l.

Os testes de suscetibilidade em discos foram realizados pelo método de Bauer-Kirby, modifi cado segundo o National Committee for Clinical
Laboratory Standards. Dois discos de imipe nem, de $10 \mu \mathrm{g}$, foram utilizados para cada cepa.

Adotou se como criterio de suscetibilidade. o seguinte padrão, sugerido por experiencia de outros autores (SHUNGU \& col.): diametro do halo de inibiçáo do crescimento $\geqslant 16 \mathrm{~mm}=$ sen sivel: 14 a $15 \mathrm{~mm}=$ intermediário; $\mathrm{e} \leqslant 13 \mathrm{~mm}$ $=$ resistente.

Paralelamente foram realizadas com as ce pas isoladas, provas de suscetibilidade, pelo mesmo método do disco, a 24 diferentes antimi crobianos de uso corrente em clínica, com pa dróes interpretativos já cstabelecidos. Foram in cluidos diariamente, a cada conjunto testado, cepas de controle de qualidade correspondentes a: Escherichia coli A TCC 25922 , Staphylococcus aureus ATCC 25923, Pseudomonas aeruginosa ATCC 27852 e Streptococcus faecalis ATCC 29212 .

\section{RESULTADOS}

Das 1230 cepas isoladas no material clinico, 1106 corresponderam a bacterias aeróbicas coriundas do Hospital das Clínicas, FMUSP; Santa Casa de São Paulo; Hospital das Clínicas da FMUFB e Hospital das Clínicas da FMUERJ । e 124 anaeróbios $(110$ provenientes do Hospital das Clínicas da FMUFRJ e 14 da Santa Casa de São Paulo). A distribuição dos patógenos ae róbios encontra se expressa na Tabela 1 e dos anaeróbios na Tabela 2 .

Todos os patógenos isolados, com exceçāo dos 110 anaeróbios oriundos da FMUFRJ foram submetidos a prova de suscetibilidade pelo mé todo do disco. Os resultados das 1120 cepas tes tadas encontram-se na Tabela 3 .

Houve boa correlaçâo do método do disco com as respectivas concentraçōes inibitórias mí nimas cuja análise global dos resultados com 1131 cepas testadas está expressa na Tabela 4 .

Os resultados das determinaçôes das con centraçōes inibitórias mínimas das 110 cepas de anaeróbios isoladas na UFRJ encontram se na Tabela 5

A correlação entre os resultados de susceti bilidade pelo método dos discos e as concen 
GODOY, C. V. F. de; MENDES, C. M. F.; MIMIC,A, I.; OLIVEIRA, M. de: SUASSUNA. I. \& UZEDA. M. de _. Estudo da suscetibilidade "in vitro" a um novo antimicrobiano (IMIPENEM) de patógenos isolados de pacientes hospitalares em vários centros. Rev. Inst. Med. trop. S. Paulo, 31(3): 169-176, 1989.

traçōes inibitórias mínimas de imipenem para as 9 espécies bacterianas isoladas com maior fre qüencia encontram-se na Tabela 6 e as respectivas variações, na Tabela 7 .

TABELA 1

Distribuição dos patógenos aeróbios isolados, por ordem de frequencia

\begin{tabular}{lclc}
\hline Microorganismo & N. de cepas & Microorganismo & N. de cepas \\
\hline Escherichia coli & 263 & Salmonella sp & 27 \\
Pseudomonas aeruginosa & 160 & Proteus sp & 17 \\
Staphylococcus aureus & 143 & Morganella morganii & 15 \\
Klebsiella pneumoniae & 102 & Citrobacter sp & 10 \\
Klebsiella sp & 83 & Staphylococcus epidermides & 8 \\
Proteus mirabilis & 76 & Streptococcus pyogenes & 7 \\
Enterobacter sp & 64 & Providencia sp & 3 \\
Streptococcus faecalis & 50 & Pseudomonas sp & 2 \\
Acinetobacter sp & 39 & Pseudomonas fluorescens & 1 \\
Serratia sp & 35 & Pseudomonas maltophilia & 1 \\
\end{tabular}

TABELA 2

Distribuição dos patógenos anaeróbios isolados, por ordem de freqüencia

\begin{tabular}{lclc}
\hline Microorganismos & N. de cepas & Microorganismos & N: de cepas \\
\hline Bacteroides fragilis & 46 & Bacteroides thetaiotaomicron & 6 \\
Clostridium difficile & 26 & Bacteroides vulgatus & 6 \\
Bacteroides sp & 14 & Bacteroides ovatus & 4 \\
Bacteroides distasonis & 8 & Bacteroides uniformis & 4 \\
Clostridium perfringens & 7 & Bacteroides melaninogenicus & 3 \\
& Total $=124$ & & \\
\hline
\end{tabular}

TABELA 3

Resultados do teste de suscetibilidade ao imipenem pelo méto do do disco para 1120 cepas de patógenos isolados

\begin{tabular}{lcr} 
& No de cepas & \multicolumn{1}{c}{$\%$} \\
\hline Sensivel (halo $\geqslant 16 \mathrm{~mm}$ ) & 1084 & 96,79 \\
Intermediário (halo de $14 \mathrm{a}$ & & \\
$15 \mathrm{~mm}$ ) & 11 & 0.98 \\
Resistente (halo $\leqslant 13 \mathrm{~mm}$ ) & 25 & 2,23 \\
Total & 1120 & 100,00 \\
\hline
\end{tabular}

Ilämetro da zona de inibicāo do crescimento $26,14 \pm 6,27$.

\section{DISCUSSÃO}

Nossos resultados permitem inferir a eficá cia "in vitro" do Imipenem contra as 1230 cepas de patógenos isolados. A suscetibilidade das 1120 cepas aeróbias do antibiótico pelo método do disco foi da ordem de $96.79 \%$, com resistência em apenas $2,23 \%$ e menos de $1 \%$ de "interme. diários" (Tabela 3 ), resultados estes comparáveis a publicações na literatura internacional

TABELA 4

Resultados globais da determinaçāo de concentraçáo inibitória minima (CIM) nas 2131 cepas testadas.

\begin{tabular}{|c|c|c|}
\hline CIM & $\mathrm{N}$ de cepas & $\%$ \\
\hline$\varsigma 4.0 \mu \mathrm{g} / \mathrm{ml}$ (sensivel) & 1044 & 92,31 \\
\hline 5 a $8,0 \mu \mathrm{g} \mathrm{ml}$ (intermediário) & 37 & 3,27 \\
\hline$=9.0 \mu \mathrm{g} / \mathrm{ml}$ (resistente) & 50 & 4,42 \\
\hline Total & 1131 & 100.00 \\
\hline
\end{tabular}

CIM $1,86 \pm 0,47$

$\mathrm{CIM}_{4,1} 0,80 \pm 1,27$ 
GODOY. C. V. F. de: MENDES, C. M. F.; MIMIÇA, I.; OLIVEIRA, M. de; SUASSUNA, I. \& UZEDA, M. de - Estudo da suscetibilidade "in vitro" a um novo antimicrobiano (IMIPENEM) de patógenos isolados de pacientes hospitalares em vários centros. Rev. Inst. Med. trop. S. Paulo, 31(3): 169 176, 1989.

TABELA 5

Concentrações inibitórias mínimas do imipenem aos patógenos anaeróbios isolados.

\begin{tabular}{lccc}
\hline Patógenos & No de cepas & CIM (mcg/ml) & $\begin{array}{c}\text { No de amostras } \\
\text { resistentes }\end{array}$ \\
\hline Bacteroides fragilis & 46 & $0,48 \pm 0,80$ & 0 \\
Bacteroides distasonis & 8 & 0,46 & 0 \\
Bacteroides thetaiotaomicron & 6 & 0,83 & 0 \\
Bacteroides vulgatus & 6 & 0,40 & 0 \\
Bacteroides ovatus & 4 & 0,50 & 0 \\
Bacteroides uniformis & 4 & 0,44 & 0 \\
Bacteroides melaninogenicus & 3 & 0,17 & $18(69 \%)$ \\
Clostridium difficile & 26 & $19,69 \pm 11,25$ & 0 \\
Clostridium perfringens & 7 & $0,30 \pm 0,31$ & $18(16,36 \%)$ \\
\hline Total & 110 & $5,01 \pm 9,89$ & 18 \\
\hline
\end{tabular}

TABELA 6

Correlaça en tre suscetibilidade ao imipenem pelo método de disco e concentraęóes inibitórias minimas para as 9 espécies bacterianas isoladas com maior frequẻncia

\begin{tabular}{|c|c|c|c|c|c|c|c|c|c|c|c|c|c|c|c|c|}
\hline \multirow[b]{3}{*}{ E. c'oli } & \multicolumn{6}{|c|}{ Diseo } & \multicolumn{6}{|c|}{$\mathrm{CIM}$} & \multicolumn{4}{|c|}{ CIM } \\
\hline & \multicolumn{2}{|c|}{ sensivel } & \multicolumn{2}{|c|}{ intermediario } & \multicolumn{2}{|c|}{ resistente } & \multicolumn{2}{|c|}{ sensivel } & \multicolumn{2}{|c|}{ intermediário } & \multicolumn{2}{|c|}{ resistente } & \multicolumn{2}{|c|}{ até $4 \mathrm{mcg} \mathrm{ml}$} & \multicolumn{2}{|c|}{ atê $8 \mathrm{mcg} \mathrm{ml}$} \\
\hline & 251 & 99.21 & 1 & 0.40 & 1 & 0.40 & 216 & 99.54 & 0 & 0.00 & 1 & 0.46 & 216 & 99,54 & 216 & 99,54 \\
\hline Pseudomonas aeruginosa & 142 & 93.42 & 3 & 1.97 & 7 & 4.61 & 116 & 78.38 & 21 & 14.19 & 11 & 7.43 & 116 & 78,38 & 137 & 92,57 \\
\hline Staphylococcus aureus & 106 & 87.60 & 0 & 0,00 & 15 & 12,40 & 105 & 86.78 & 1 & 0.83 & 15 & 12.40 & 105 & 86.78 & 106 & 87,60 \\
\hline Bacteroides $s p$ & & & & & & & 77 & 100.00 & 0 & - & 0 & - & 77 & 100,00 & 77 & 100.00 \\
\hline Klebsiella pneumoniae & 101 & 100.00 & 0 & 0,00 & 0 & 0.00 & 102 & 100,00 & 0 & 0.00 & 0 & 0.00 & 102 & 100.00 & 102 & 100.00 \\
\hline Klebsiella sp & 79 & 98.76 & 0 & 0.00 & 1 & 1.24 & 55 & 98.21 & 0 & 0.00 & 1 & 1.79 & 55 & 98,21 & 55 & 98.21 \\
\hline Proteus mirabilis & 71 & 97,26 & 2 & 2.74 & 0 & 0.00 & 62 & 86.11 & 9 & 12.50 & 1 & 1.39 & 62 & 86.11 & 71 & 98,61 \\
\hline Enterobacter sp & 48 & 94,12 & 3 & 5.88 & 0 & 0.00 & 46 & 100.00 & $\therefore$ & 0.00 & 0 & 0.00 & 46 & 100,00 & 46 & 100,00 \\
\hline Streptococcus faecalis & 50 & 100,00 & 0 & 0.00 & 0 & 0.00 & 50 & 100.00 & 0 & 0.00 & 0 & 0.00 & 50 & 100,00 & 50 & 100,00 \\
\hline
\end{tabular}

TABELA 7

Médias dos diâmetros de halos de inibição pelo método do disco e concentraçōes inibitórias mínimas e respectivos desvios para as 9 espécies bacterianas isoladas com maior freqüencia.

\begin{tabular}{lccc}
\hline & DISCO & CIM & CIM $_{90}$ \\
\hline E. coli & $25.29 \pm 4,10$ & $0,24 \pm 0,65$ & $0,16 \pm 0,14$ \\
Pseudomonas aeruginosa & $24,61 \pm 5,29$ & $3,91 \pm 7,97$ & $2,74 \pm 2,37$ \\
Staphylococcus aureus & $31,33 \pm 12,65$ & $2,28 \pm 6,41$ & $1,06 \pm 2,75$ \\
Bacteroides sp & & $0,48 \pm 0,65$ & $0,39 \pm 0,28$ \\
Klebsiella pneumoniae & $26,40 \pm 2,89$ & $0,34 \pm 0,39$ & $0,30 \pm 0,31$ \\
Klebsiella sp & $24,96 \pm 3,75$ & $1,44 \pm 8,32$ & $\mathbf{0 , 3 1} \pm 0,29$ \\
Proteus mirabilis & $22,70 \pm 3,44$ & $2,04 \pm 1,92$ & $1,87 \pm 1,46$ \\
Enterobacter sp & $24,63 \pm 3,83$ & $0,80 \pm 0,88$ & $\mathbf{0 , 6 7} \pm 0,55$ \\
Streptococus faecalis & $24,14 \pm 3,48$ & & $0,70 \pm 0,47$ \\
\hline
\end{tabular}


GODOY, C. V. F. de; MENDES, C. M. F.; MIMIÇA, I.; OLIVEIRA, M. de; SUASSUNA, I. \& UZEDA, M. de - Estudo da suscetibilidade "in vitro" a um novo antimicrobiano (IMIPENEM) de patógenos isolados de pacientes hospitalares em vários centros. Rev. Inst. Med. trop. S. Paulo, $31(3)$ : 169-176, 1989

(KROPP \& col., $1985 ;$ SHUNGU \& col.; WILLIAMS; WEXLER \& FINEGOLD).

Configura-se a eficácia como muito favorá vel, sobretudo se considerarmos tratar-se nosso material de patógenos recém-isolados, agentes de infecçōes bacterianas em pacientes, em sua maioria hospitalizados, portadores em geral, de elevados niveis de resistência à maioria dos antimicrobianos. As cepas testadas foram predominantemente de' gram negativos, com pouca sensibilidade aos demais antibióticos, destacandose em nosso material a Escherichia coli, Enterobacter sp, Acinetobacter sp, Salmonella sp e Serratia sp. A suscetibilidade das cepas de Pseudomonas sp (todas Pseudomonas aeruginosas, com exceção de 2) pela prova do disco, da ordem de $93,42 \%$ foi extraordinária, mas as poucas ce pas resistentes apresentavam correspondência de concentraçōes inibitórias mínimas muito elevadas. A única cepa de Pseudomonas fluorescens incluída mostrou-se sensivel, e a remanescente, correspondente à Pseudomonas maltophilia apresentou resistência. Houve boa correspondència entre os resultados da suscetibilidade ao imipenem pela prova do disco e as respectivas concentraçōes inibitórias mínimas do antibiótico aos vários patógenos, obtendo-se $92,31 \%$ de resultados inferiores ou iguais a $4,0 \mu \mathrm{g} / \mathrm{ml} \mathrm{e}$ $95,59 \%$ inferiores ou iguais a $\mathbf{8 , 0} \boldsymbol{\mu \mathrm { g }} / \mathrm{ml}$, com CIM médio de $1,86 \pm 0,47$ e $\mathbf{C I M}_{90} 0,80 \pm 1,27$ (Tabela 4). A boa correspondência entre o método do disco e as determinações do CIM permite pois o emprego rotineiro do primeiro, mais difundido em laboratórios de Patologia Clínica, para avaliaçāo da suscetibilidade bacteriana ao antibiótico.

Entre os gram-positivos destaca-se a suscetibilidade $(100 \%)$ do Streptococcus faecalis ao imipenem com perfeita correspondência de CIM (Tabela 6). As cepas de Staphylococcus aureus, predominantes entre os gram-positivos em nos so material, apresentaram $87,60 \%$ de suscetibilidade e $12,4 \%$ de resistência pelo método do disco com perfeita concordância das concentrações inibitórias mínímas $(86,78 \% \leqslant 4,0 \mu \mathrm{g} / \mathrm{ml}$ e $12,40 \%$ $>\mathbf{8 , 0} \mu \mathrm{g} / \mathrm{ml}$ ). Convém ressaltar que as cepas resistentes, hospitalares, apresentaram simultaneamente resistência à oxacilina, e inversamen te, praticamente todas as cepas resistentes à oxacilina o foram ao imipenem. Por estes resul tados, nāo se configura o antibiótico como alternativa para a terapêtica de infecçôes por Staphylococcus aureus oxacilina-resistentes.

Com os patógenos anaeróbios, principalmente cepas do gènero Bacteroides sp (Tabela 5) os resultados das concentraçōes inibitórias mínimas ao imipenem foram plenamente satis fatórios, todos com valores iguais ou inferiores a $4 \mu \mathrm{g} / \mathrm{ml}$. Com o gênero Clostridium sp, igual resultado foi obtido com as 7 cepas de Clostridium perfringens testadas, mas entre as $26 \mathrm{ce}$ pas de Clostridium difficile incluidas, 18 (69\%) apresentaram concentraçōes inibitórias mínimas superiores a $\mathbf{8 , 0} \boldsymbol{\mu} \mathrm{g} / \mathrm{ml}$, consideradas resis tentes, resultados estes concordantes com outros autores (WILLIAMS; WEXLER \& FINE. GOLD).

Nossos resultados permitem sugerir que o imipenem é antibiótico eficaz contra grande nú mero de diferentes patógenos bacterianos. Pare ce-nos pois trazer real contribuição à terapêutica das infecçōes, considerando-se sobretudo eventualidades de infecçōes mistas por aeróbios e anaeróbios e para a terapêtica inicial presun tiva em infecçōes graves.

\section{CONCLUSÕES}

Configura-se, pelos resultados apresentados, possuir o imipenem boa atividade "in vitro" contra os patógenos mais freqüentemente isolados, destacando-se sua atividade para gram-negativos com elevados niveis de resistência a grande número de antimicrobianos (Pseudomonas sp, Serratia sp e Enterobacter sp entre ou tros), gram-positivos (Streptococcus faecalis e Staphylococcus aureus oxacilina-sensíveis) e anaeróbios (particularmente Bacteroides $\mathrm{sp}$ ). As provas de suscetibilidade ao imipenem de 1230 cepas de patógenos recém-isolados de pacientes com infecçōes variadas, na maioria hospitalizados, mostraram-se plenamente satisfatórias com $96,79 \%$ de suscetibilidade pela prova do disco e com boa correspondencia pela determinaçáo das concentraçōes inibitórias mínimas do antibiótico: CIM médio de $1.86 \mu \mathrm{g} / \mathrm{ml}$ com desvio de $\pm 0,47$ e CIM $_{90}$ de $0,80 \mu \mathrm{g} / \mathrm{ml}$ com desvio de $\pm 1,27$ 
GODOY, C. V. F. de: MENDES, C. M. F.: MIMIÇA, I.; OliveirA, M. de; SUAssunA, I. \& UZEDA, M. de - Estudo da suscetibilidade "in vitro" a um novo antimicrobiano (IMIPENEM) de patógenos isolados de pacientes hospitalares em vár us centros. Rev. Inst. Med. trop. S. Paulo, 31(3): 169-176, 1989

\section{SUMMARY}

"In vitro" efficacy of a new antimicrobial agent (IMIPENEM) in pathogens isolated from hospitalized patients from several Medical Centers in Brazil.

Imipenem is a $\boldsymbol{\beta}$ lactam antibiotic, a highly potent new carbapenem with broad antibacterial spectrum. To test the "in vitro" efficacy of this antimicrobial agent in pathogens more fre quent in several Medical Centers in Brazil, susceptibility testing with $10 \mathrm{mcg}$ imipenem disks and, or corresponding MIC were carried out with 1231 recent isolates of 41 different bacterial species, obtained mainly from hospitalized patients in 5 different medical centers of the cities of $\mathbf{S}$. Paulo, Rio de Janeiro and Salvador. Our preliminary results with this antibiotic, in final phases of clinical and laboratorial experimentation in this country, are very promissing with, $96.79 \%$ of susceptibility of test isolates to $10 \mathrm{mcg}$ imipe nem disks and corresponding MIC correlation of up to $4 \mu \mathrm{g} / \mathrm{ml}$. $(92.31 \%)$.

Of the 9 bacterial species more frequently isolated totaling $1108(82 \%)$ of the 1230 test isola tes, disk susceptibility was $99 \%$ (E. coli), 93\% (Pseudomonas aeruginosas), $87 \%$ (Staphylococcus aureus), $100 \%$ (Klebsiella pneumoniae), $98 \%$ (Klebsiella sp), 97\% (Proteus mirabiles), 94\% (Enterobacter $\mathrm{sp}$ ), 100\% (Streptococcus faecalis) with good MIC correlation (up to $8 \mathrm{mcg} / \mathrm{ml}$ ) and $100 \%$ for the anaerobic species Bacteroides sp (MIC up to $4 \mu \mathrm{g} / \mathrm{ml}$ ).

"In vitro" efficacy to hospital pathogens with high frequency of resistance to most antibiotics as Pseudomonas aeruginosa and to anaerobes notably Bacteroides $\mathrm{sp}$ is emphasized

\section{AGRADECIMENTO}

Os autores agradecem à Bioquímica Farmacêtica Neusa Augusta de Oliveira Mazieri e à Estaticista Paula Goldenstein Strassmann pela colaboração prestada.
REFERÊNCIAS BIBLIOGRÁFICAS

1. ALBERS-SCHONBERG, G.; ARISON, B. H.;HENSENS O. D.: HIRSCHFIELD, J.; HOOGSTEEN, K.; KACZKA, E. A. RHODES, R. E. KAHAN, J. S.; KAHAN, F. M RATCLIFFE, R. W.; WALTON, E.; RUSWINKLE, L. J ; MORIN, R.B. \& CHRISTENSEN, B. G. - Structure and absolute configuration of thienamycin. J. Amer. chem. Soc., 100: 6491-6499, 1978.

2. BARRY, A. L. \& THORNSBERRY, C. - Susceptibility testing: diffusion test procedures. In: LENNETTE, E. H.; BALOWS, A.; HAUSLER, J. B. \& SHADOMY, H. J. - Manual of clinical microbiology. 4th ed. Washington, Ame rican Society for Microbiology, 1985. p. 463.474

3. BARRY, A. L.; THORNSBERRY, C.: GAVAN, T. L. \& JONES, R. N. - Interpretive standards and quality con trol guidelines for imipenem susceptibility test with 10 meg disks. J. clin. Microbiol., 20: 988-989, 1984

4. KAHAN, J. S.; KAHAN, F. M.; GOEGELMAN, R.; CUR RIE, S. A,; JACKSON, M.; STAPLEY, E.D,; MILLER, T W.; MILLER, A. K.; HENDLIN. D.: MOCHALES, S.: HER NANDEZ, S : WOODENFF, H. B. \& BIRNBAUM, J.Thienamycin, a new betalactam antibiotic I. Discovery taxonomy, isolation and physical properties. J. Antibiot. (Tokyo), 32: 1 13, 1979

5. KESADO, T ; HASHIZUME, T. \& ASAHI, Y. - Antibac terial activities of a new stabilized thienamycin, $\mathbf{N}$-formi midoyl Thienamycin in comparison with other antibio tics. Antimicrob. Agents Chemother, 17: $912.917,1980$

6. KROPP, H.; GERCKENS, L.; SUNDELOF, J. G. \& KA HAN, F. M. - Antibacterial activity of imipenem: the first thienamycin antibiotic. Rev. infect. Dis., 7 (suppl. 3 ) $389410,1985$.

7. KROPP, H.; SUNDELOF, J. G ; KAHAN, J. S.; KAHAN F. M. \& BIRNBAUM, J. - MK 0787 (N-formimidoy 1 thie namycin in comparision with other antibiotics. Antimicrob. Agents Chemother, 17: 993-1000, 1980.

8. LEANZA, W. J.; WILDONGER, K. J.: MILLER, T. W. \& CHRISTENSEN, B. G. - N acetimidoy 1 and $\mathbf{N}$-formimi doy 1 thienamycin derivatives; antipseudomonal beta-lac tam antibiotics. J. medic. Chem., 22: 1435-1436, 1979

9. NATIONAL Committee for Clinical Laboratory Stan dards - Performance Standards for antimicrobial disk susceptibility test. Approved Standard M2-A3 National Committee for Clinical Laboratory Standards. Villanova. Pa., 1984.

10. NATIONAL Committee for Clinical Laboratory Stan dards - Methods for dilution antimicrobial susceptibility test for bacteria that grow aerobically. Candidate Stan dard for approval. M7 -A. National Committee for Clinical Laboratory Standards. Villanova., Pa., 1985.

11. SHUNGU, D. L.; CERAM1, A. T.; WEINBERG, E.; CAPIZ ZI, T. \& GADEBUSCH, H. H. - Tentative interpretive criteria for "in vitro" antibacterial susceptibility testing with Imipenem. J. clin. Microbiol., 23 421-424, 1986. 
GODOY, C. V. F. de: MENDES, C. M. F.; MIMICA, I.; OLIVEIRA, M. de; SUASSUNA, I. \& UZEDA, M. de - Estudo da suscetibilidade "in vitro" a um novo antimicrobiano (IMIPENEM/ de patógenos isolados de pacientes hospitalares em vários centros. Rev. Inst. Med. trop. S. Paulo, 31(3): 169 176, 1989

12. SUTTER, V.L. \& WASHINGTONII, $\boldsymbol{J}$. A. - Susceptibility testing of anaerobes. In: LENNETTE, E. H,; BALOWS, A.; HAUSLER JR., W. \& SHADOMY, H. J. - Manual of clinical microbiology. 4 th ed. Washington. American Suciety for Microbiology, 1984. Chapter 45, p. 475.477.

13. WASHINGTON II, J \& SUTTER, V. L. - Dilution suscep tibility test: agar and macro - Broth dilution procedures In LENNETTE, E. H,; BALOWS, A, HAUSLER JR., W \& SHADOMY. H. J. - Manual of clinical microbiology. 4th ed. Washington. American Society for Microbiology. 1984. Chapter 42, p. $453-458$
14. Williams, J . D. - Activity of Imipenem against Pseudomonas and Bacteroides species. Rev. infect. Dis., 7 (suppl. 3 เ: 411416,1985

15. WEXLER, H. M. \& FINEGOLD, S. M. -- "In vitro" activity of imipenem against anaerobic bacteria. Rev. infect. Dis., 7 (Suppl.3): 417-425, 1985.

Recebido para publicação em 13/7/1988 